\section{Photo atlas of inner skull base using digital single lens reflex camera}

Sir,

Cadaver dissection has a pivotal role in shaping the initial learning curve of skull base neurosurgeons. At present, cadaver dissection is not formally practiced in India barring a few examples. It is limited to some conferences and research projects only. The reasons for the same are many including limited availability of cadavers, poor facilities for cadaver procurement and preservation, inadequate infrastructure, and financial constraints to name a few. Operating microscope is must for capturing high-resolution images. Due to nonavailability of a dedicated cadaver laboratory, we developed atlas of inner skull base using digital single lens reflex (DSLR) camera. A prior permission from institute's authority was taken to conduct this study. The study was done in mortuary, where clinical and medicolegal autopsies are conducted. The current study was limited to exposure of fifth (V) and sixth (VI) cranial nerves. Right side of head of a fresh cadaver was used for dissection. The temporal bone was removed until middle cranial fossa base. The dura was stripped and interdural dissection was done to expose greater superficial petrosal and V nerves. After removal of brain and tent, VI nerve was traced from origin to Dorello's canal. The Gruber's ligament was cut, dura opened over cavernous sinus, and VI nerve was traced till superior orbital fissure. Finer anatomy such as cranial nerve pathway and relationship of the anterior clinoid process could also be adequately demonstrated.

The following gears were used for capturing the images: Cannon 550D ${ }^{\circledR}(18-55 \mathrm{~mm} \mathrm{1:3.5-5.6} \mathrm{IS)}$ and Nikon D100 ${ }^{\circledast}(60 \mathrm{~mm})$. The images were further edited with Adobe Photoshop CS2 ${ }^{\circledR}$. The images captured are

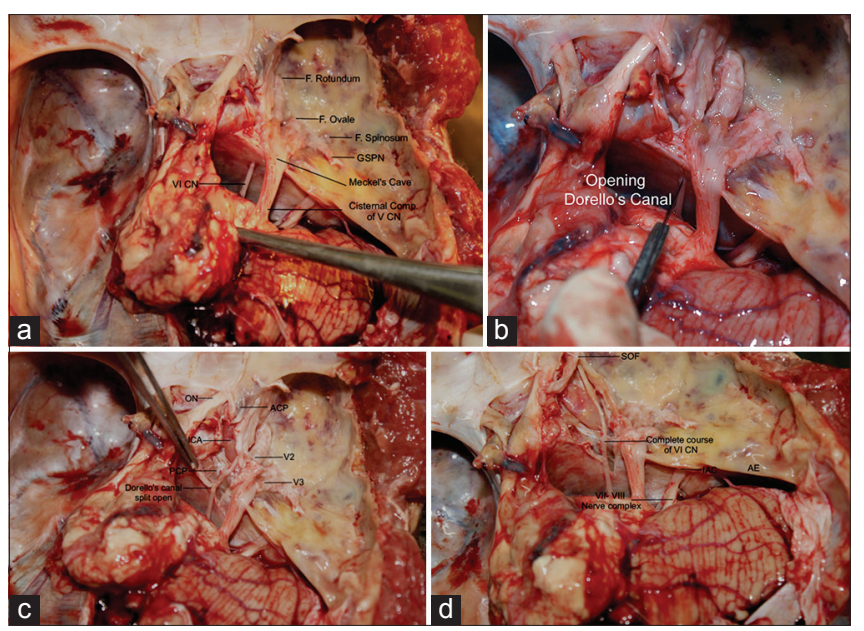

Figure 1: (a) Posterior fossa showing cranial nerves, (b) opening of dorello's canal, (c) sixth nerve in cavernous sinus, (d) complete course of sixth nerve 


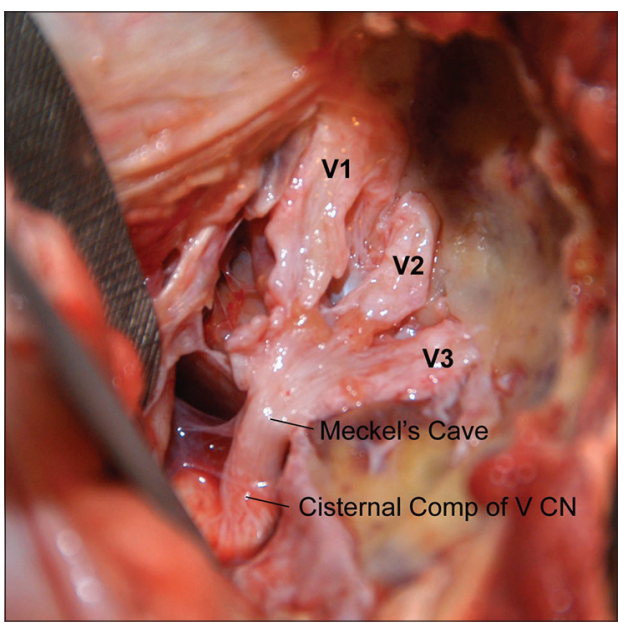

Figure 2: Fifth nerve and its divisions

of high-quality and comparable to images obtained with operating microscope [Figures 1 and 2].

We use these photographs for teaching and training residents. These images when placed in a stepwise manner help in revising the complex neuroanatomy especially at the remote skull base locations. The limitation was that the blood vessels were not infused, hence not distinctly visualized. The images can be of better educational purposes with the availability of injected heads with demonstration of cerebrovascular structures.
The very basic motto of this attempt lies in the fact that the complexities of neuroanatomy and neurosurgical procedures can be demonstrated at any medical institute without the need of sophisticated and costly cadaver dissection set up and DSLR cameras can be used to take good quality photographs of skull base for educational purpose.

Manjul Tripathi, Dhananjaya I. Bhat, Dhaval P. Shukla

Department of Neurosurgery, National Institute of Mental Health and Neurosciences, Bengaluru, Karnataka, India

Address for correspondence: Dr. Dhaval P. Shukla, Department of Neurosurgery, National Institute of Mental Health and Neurosciences, Bengaluru, Karnataka, India. E-mail: neurodhaval@rediffmail.com

\begin{tabular}{|l|l|}
\hline \multicolumn{2}{|c|}{ Access this article online } \\
\hline Quick Response Code: & Website: \\
\hline & www.ruralneuropractice.com \\
\cline { 2 - 3 } & \\
\hline & \\
\hline
\end{tabular}

